\title{
Intracranial abscess formation in an adult alpaca: a case report
}

\author{
Sonja Franz ${ }^{*}$ (D), Sandra Högler ${ }^{2}$, Michaela Gumpenberger ${ }^{3}$ and Agnes Dadak ${ }^{4}$
}

\begin{abstract}
Background: Intracranial abscess formation is an extremely rare and sporadically documented disease in South American Camelids (SACs). Herein we report the first case of otogenic brain abscess formation in this species.

Case presentation: A 4 years old female alpaca was presented to our veterinary hospital with a 6 month history of neurologic disorder symptoms, mainly head tilt to the right and emaciation. A comprehensive workup (ultrasound and computed tomography) revealed irreversible cranial nerve abnormalities, extensive lesions in the region of external, middle and internal right ear including destruction of bony structures (tympanic bulla, parts of temporal bone) and severe brain deformation caused by an intracranial abscess. The lesion was up to $6 \times 7 \times 4 \mathrm{~cm}$ and occupying almost $40 \%$ of the cranial cavity. No pathological findings were evident in other organs or structures. The late referral of the alpaca at this advanced stage of destructive disease precluded surgical intervention.

Conclusions: This case report describes the clinical signs, diagnostic procedures and pathological findings in an adult female alpaca suffering from cranial nerve abnormalities caused by a massive otogenic brain abscess. Camelids suffering from otitis may not present with clinical signs until the pathology is severe. The importance of considering intracranial abscess formation as differential diagnosis in SACs showing the merest hint of nerve deficits cannot be emphasized enough in order to diagnose such pathological processes at an early and treatable stage.
\end{abstract}

Keywords: South American camelids, Brain, Abscess, Otitis, Computed tomography, Pathology, Neurologic disease

\section{Background}

In South American camelids (SACs) neurologic disorders caused by infectious or non-infectious agents are not uncommon [1-3], albeit there are no epidemiologic studies published on the incidence of these diseases so far. Besides, only a few case reports are available providing insight into aetiology, diagnosis, treatment, and outcome of neurologic camelid patients [1-6].

In SACs brain abscesses causing neurologic symptoms usually occur in response to bacterial infections. They either develop through hematogenous spread of bacteria from other anatomical sites or they result from direct extension of cranial infections [2,3]. Clinical signs can vary depending on the region of the brain affected. To the author's knowledge, only three case reports are published on intracranial abscess formation in SACs [4-6]. Brain abscesses were mainly found in crias and

\footnotetext{
*Correspondence: Sonja.Franz@vetmeduni.ac.at

${ }^{1}$ Clinic for Ruminants, Clinical Unit of Ruminant Medicine, University of

Veterinary Medicine Vienna, Veterinärplatz 1, 1210 Vienna, Austria

Full list of author information is available at the end of the article
}

described as being an important complication of failure of passive transfer. The formation of intracranial abscesses was reported once in a 2-week-old alpaca cria caused by E. coli [4] and another time in a 1-month-old alpaca cria caused by Fusiformes spp. infection [6]. A potential microbial dissemination originating from a molar abscess in the mandible was considered to be causative for development of brainstem and cerebellum abscessation in an 11 years old male alpaca [5].

To our knowledge, this is the first description of intracranial abscess formation in SACs occurring as a complication from otitis rather than hematogenous spread from another site of infection or from cranial infection. This case report describes the clinical signs, diagnostic procedures and pathological findings in an adult female alpaca suffering from cranial nerve abnormalities caused by a massive otogenic brain abscess.

\section{Case presentation}

A 4 years old female alpaca was referred to the clinic for ruminants, University of Veterinary Medicine Vienna,

(c) The Author(s). 2019 Open Access This article is distributed under the terms of the Creative Commons Attribution 4.0 International License (http://creativecommons.org/licenses/by/4.0/), which permits unrestricted use, distribution, and 
Austria. The alpaca had a 6 month history of neurologic disorder signs. The main clinical signs were head tilt to the right and emaciation (Fig. 1a). The owner reported that he had treated the alpaca subcutaneously against mites several months before referral and that he had observed a slow deterioration of the clinical signs within the last 3 months. The alpaca separated from the herd but never showed inability to feed or dislike of the feeding. The alpaca was kept with other alpacas on a pasture, and all animals had access to additional feed such as hay and mineral supplements. No other alpacas on the farm were affected. The alpaca had given birth to a healthy cria 1 month before referral to the clinic.

On initial physical examination the female alpaca showed a quiet behavior, the body weight was $46.2 \mathrm{~kg}$. Heart rate and respiratory rate were within reference ranges. The rectal temperature was $37.8^{\circ} \mathrm{C}$. Auscultation of lungs and heart was uneventful. No abnormalities

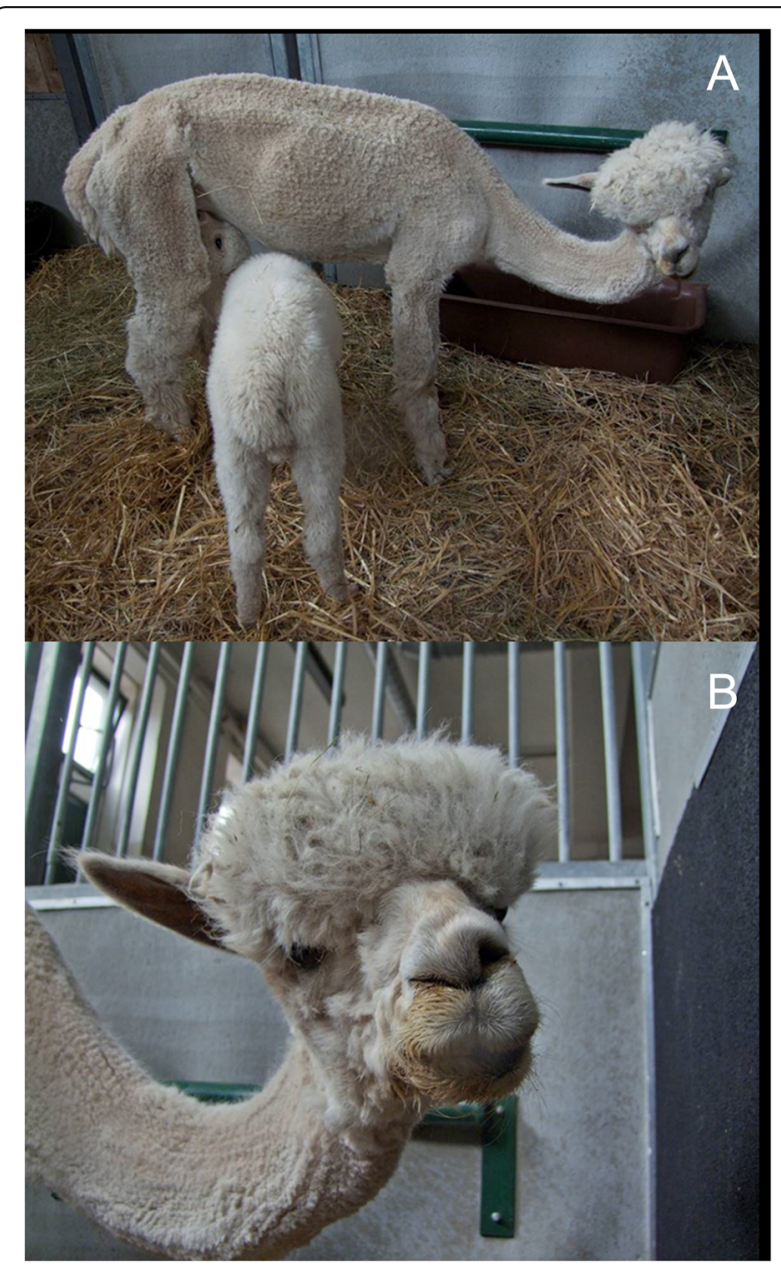

Fig. 1 Adult female alpaca with its cria on initial physical examination. a the alpaca is emaciated and shows a lowered neck with head tilt to the right. $\mathbf{b}$ Collapse of the right nostril and ipsilateral lip droop were detected at abdominal palpation and auscultation. On both forelimbs the skin between the toes was hairless, thickened and crusty.

A neurologic examination was performed to determine the region of the nervous system being most likely affected.

Postural assessment of the alpaca revealed a lowered neck with a head tilt to the right and an ipsilateral lip droop with small amounts of cud dropping from the affected side. However, the animal was able to prehend food, chew and swallow. Further, collapse of the right nostril and deviation of the nasal philtrum to the left was observed (Fig. 1b). Atrophy of the muscles of mastication on the right side was diagnosed by palpation.

Additionally a plum-sized solid swelling at the base of the right ear was palpable. While the left ear was held in vertical position and moved constantly in response to sound, the right ear was held horizontally, never changing its position. No movement of the pinna occurred when testing the sensitivity of the right ear canal. There were no visible signs of trauma, alopecia or inflammation at the external pinna of the right ear.

In addition the alpaca showed loss of the menace reflex on the right side where the corneal as well as palpebral reflexes were impaired in contrast to the left side where immediate closure of the eyelid could be induced. Moreover, the alpaca was able to pass obstacles in a normal manner implying absence of significant visual impairment.

Observational gait analysis showed no ataxia or circling. Postural reaction (limb correction of awkward position) and spinal reflexes (withdrawal reflex, perineal reflex) were unremarkable.

In summary, neurological examination revealed multiple cranial nerve deficits. Dysfunction of the trigeminal nerve was linked to the observed atrophy of the masticatory muscle. Facial nerve defect was mainly responsible for pathological impaired corneal, palpebral and menace reflexes. Additionally, the defect of the facial nerve could have been causative for asymmetry of nose and lips, and even immobility of the right pinna, since no reflex could be induced by sensitivity testing. Dysfunction of the vestibulocochlear nerve resulted in head tilt.

Based on the neurological deficits identified by physical examination of this alpaca (cranial nerve deficits evident, no involvement of spine and limbs) the primary differential diagnosis included otitis media and interna.

For further diagnostic purposes, blood cell count was analyzed and showed a mild regenerative anemia (RBC $9.9710^{12} / \mathrm{L}, \mathrm{Hb} 6.5 \mathrm{mmol} / \mathrm{L}, \mathrm{MCV} 31.9 \mathrm{fl}, \mathrm{MCH} 6.5$ $\mathrm{fmol} / \mathrm{L}, \mathrm{MCHC} 20.5 \mathrm{mmol} / \mathrm{L}$ ). Blood gas analysis and biochemistry profile were within reference ranges [7]. PCR analysis of whole blood was negative for Cand. Mycoplasma haemolamae. Parasitological examination 
of feces showed mild infection with Coccidia spp. Skin biopsies of the area between the toes of the forelimbs was tested positive for Chorioptes spp.

Otoscopic examination of the right external ear canal was performed, using a flexible endoscope with a diameter of $3.8 \mathrm{~mm}$ (Karl Storz Endoskop Austria GmbH). It was noticeable that the endoscope could be inserted into the external vertical ear canal for just about $0.5 \mathrm{~cm}$, further insertion of the endoscope was not possible due to ear canal stenosis. The visible part of the external ear canal showed endoscopically no signs of inflammation and no pathological content (e.g. foreign body or parasites).

Ultrasound examination of the swelling at the base of the right ear was performed using a $7.5 \mathrm{MHz}$ linear transducer and alcohol (ethanol 70\%) as contact medium between probe and skin. At the area of interest a spheric inhomogenic hypoechogenic structure with small echoic spots was seen (loss of normal connective tissue and muscle architecture), sonographically resembling an inflamed tissue [8]. No cranial bone could be visualized in this area.

Analysis of cerebrospinal fluid (CSF) is one of the most commonly performed ancillary diagnostic tests when investigating the cause of a neurologic disease. Changes in protein concentration, cell count, and cell differentiation can help to distinguish between inflammatory/infectious and non inflammatory/non infectious diseases [2]. Nevertheless, in this case, CSF analysis was not considered, since physical examination implicated pathological changes of the middle and inner ear structures. Although there is limited information on the use of computed tomography (CT) to visualize brain and skull alterations in SACs $[9,10]$, the decision was made to perform a CT scan, since this imaging technique has been shown to be very effective in locating spaceoccupying lesions and pathological changes in brain tissue of other animal species.

CT examination (multi-slice helical CT, Siemens Somatom Emotion using $80 \mathrm{mAs}, 130 \mathrm{kV}$, rotation time $1.5 \mathrm{~s}$, pitch 0.8 , and slice thickness $0.75 \mathrm{~mm}$ ) took place at the Clinical Unit of diagnostic Imaging, University of Veterinary Medicine Vienna. The alpaca was examined under general anesthesia in sternal recumbency, using butorphanol $(0.2 \mathrm{mg} / \mathrm{kg} \mathrm{IM})$ and xylazine $(0.4 \mathrm{mg} / \mathrm{kg} \mathrm{IM})$ as premedication, followed by maintenance medication with ketamine $(5 \mathrm{mg} / \mathrm{kg} \mathrm{IM})$ and isoflurane in oxygen (Fig. 2).

At CT examination (Fig. 3 a-c, 4A) the right osseous external ear canal was not apparent. A large spaceoccupying mass was replacing the right external acoustic meatus, and osseous ear canal, the area of the former tympanic bulla, tympanic cavity and inner ear. Remnants of the bulla wall were thickened and sclerotic. The

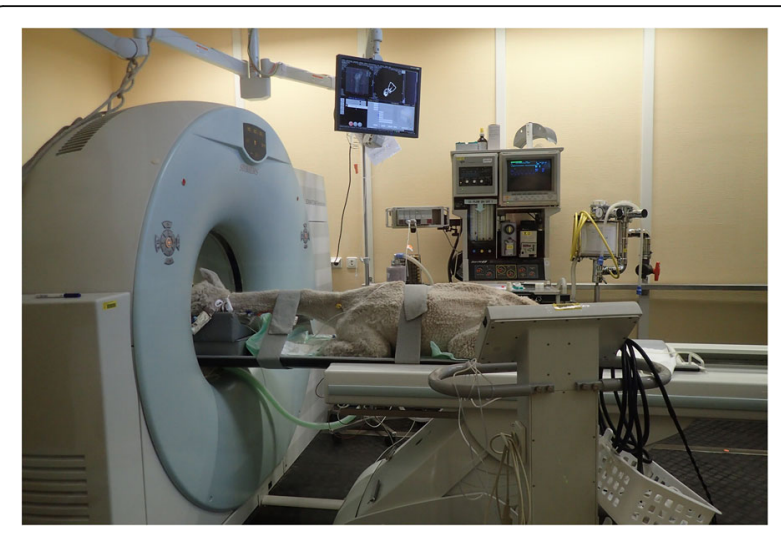

Fig. 2 Positioning of the adult alpaca in sternal recumbency for $C T$ examination of the head

ventral part of the tympanic bulla showed increased density with loss of all aerated areas of its usually honeycomb-like structure. The temporal bone showed thickening and intracranial irregular periosteal reactions dorsal to the right temporomandibular joint. The visible mass lesion was up to $6 \times 7 \times 4 \mathrm{~cm}$ (height $\mathrm{x}$ length $\mathrm{x}$ width) and was therefore occupying nearly $40 \%$ of the cranial cavity. The mass was mildly hyperintense with multiple, somehow onion shell-like calcified areas. No capsule was found. Both lateral cerebral ventricles were moderately enlarged, more on the right than on the left side. Marked midline-shift to the left was seen, causing complete compression of the caudal part of the right cerebral ventricle. The cerebellum was partially displaced into the great foramen of occipital bone resulting in a mild cerebellar herniation. Mild atrophy of the right temporal muscle was visible. The lymph nodes in the upper neck region were unremarkable.

Due to the poor prognosis, the owners decided to have the alpaca euthanized.

A full necropsy was performed at the Institute of Pathology, University of Veterinary Medicine Vienna. The skull was cut into coronary sections by a diamond saw. Samples of brain, skull and organs were fixed in $4 \%$ buffered formaldehyde solution. Samples containing bone were decalcified for $12 \mathrm{~h}$ in Decal ${ }^{\circ}$ (quartett $\mathrm{GmbH}$, Berlin, Germany). All samples were then embedded in paraffin wax. Sections of $1.5 \mu \mathrm{m}$ thickness were cut and stained with hematoxylin and eosin (HE) for histological examination. Masson's trichrome staining was performed to demonstrate collagenous fibers in the abscess capsule and Brown and Brenn staining was performed to detect gram positive and negative bacteria. A primary antibody against smooth muscle actin (SMA, mouse monoclonal antibody, no: M0815, dilution 1:1500, Agilent Technologies Österreich $\mathrm{GmbH}$, Austria) was used to demonstrate vessels and a primary antibody against glial fibrillary acidic protein (GFAP, rabbit polyclonal 


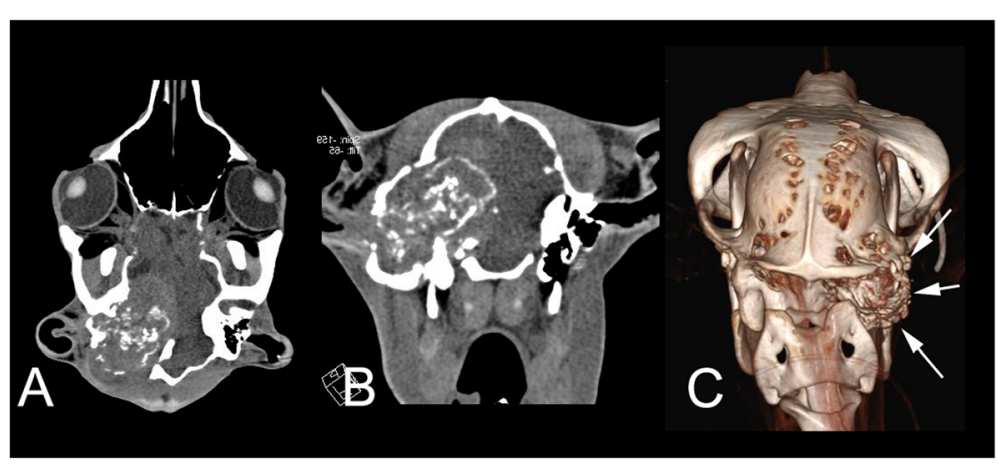

Fig. 3 Coronal (a) and transverse (b) CT image of the head of the alpaca (soft tissue window). The right side of the animal is projected on the left side of the image. A partially mineralized soft-tissue mass originated at the level of the right ear and caused severe destruction of the temporal bone, midline shift of the brain and hydrocephalus (not seen on these planes). c shows a bony 3D-model of the head and first cervical vertebra of the same animal, caudal view

antibody, no: Z0334, dilution 1:3000, Agilent Technologies Österreich $\mathrm{GmbH}$, Austria) was used to identify astrocytes in brain sections by immunohistochemistry (IHC).

On gross examination the animal was cachectic. An asymmetry of the skull was evident after the skin was removed. A mild swelling of firm consistency of the right base of the skull and discoloration and atrophy of the lateral portion of the right cerviculoscutular muscle were present. Coronary sections of the skull and brain showed a light brown-whitish gelatinous to firm mass of up to 7 $\mathrm{cm}$ in diameter replacing bone and muscle of the right cranium and expanding into the cranial cavity. The mass had a thin capsule of fibrous tissue (Fig. $4 \mathrm{~b}$ ). It encompassed the right middle and inner ear and occluded the right ear canal. The right tympanic bulla was completely destroyed in contrast to the normal left tympanic bulla (Fig. 4a, b). The mass caused severe midline shift in the caudal brain and displaced medulla oblongata, cerebellum, mesencephalon, and the caudal part of the right hemisphere to the left (Fig. 4b). The right hippocampus and thalamus were displaced cranially. A mild hydrocephalus of the lateral ventricles was evident due to obstruction of the mesencephalic duct.

Histologically the mass turned out to be an abscess with a great amount of necrotic debris in the center, followed by a layer of degenerated neutrophils adjacent to a capsule of granulation tissue and mature connective tissue (Fig. 4c). Mild to moderate infiltration of the outer layers of the capsule with lymphocytes and plasma cells was present and collagenous fibers were detectable by Masson's trichrome staining (Fig. 4c). Within the necrotic debris degenerate bone fragments were present, while bone adjacent to the abscess capsule was resorbed by osteoclasts. Focally in the temporal bone, irregularly arranged osteoid was surrounded by osteoblasts and embedded in fibrous tissue adjacent to bone. Areas of bone resorption by osteoclasts were noted. In the neuropil adjacent to the abscess severe astrogliosis was detectable. Acute neuronal degeneration was not detectable, but regions with gliosis showed a marked loss of neurons (Fig. 4d). The neuropil was edematous and showed small foci of malacia in gray and adjacent white matter. Atrophy of the cerebral cortex by pressure was evident in the right occipital lobe directly adjacent to the abscess. In some regions vascular proliferation and infiltration of vessels from the abscess capsule into the neuropil as well as mild perivascular infiltration with lymphocytes were present (Fig. 4d, e). By immunohistochemistry severe diffuse astrogliosis was detectable in brain regions adjacent to the abscess (Fig. 4f). No bacteria were detectable histologically by Brown and Brenn staining in the brain abscess.

\section{Discussion and conclusions}

In a patient suffering from a brain abscess of the size detected in this female alpaca a wider and more obvious range of clinical signs could have been expected than actually being observed in the presented case. Therefore, the importance of thorough physical and neurological examination in SACs showing the merest hint of nerve deficits cannot be emphasized enough in order to diagnose chronic processes at an early stage.

In general, differential diagnoses for cranial nerve deficits include otitis media/interna, trauma, listeriosis, aberrant parasite migration and brain abscess or tumor $[1-4,6]$.

Beside physical examination several different diagnostic approaches are available. To detect inflammatory processes of the CNS, the analysis of cerebrospinal fluid (CSF) in regard to pleocytosis and increased protein concentration is helpful $[2,3]$, but it needs to be considered that sampling of CSF requires sedation of the animal and is a risky procedure. In this case CSF analysis was not considered, since physical examination and the obvious cranial nerve deficits implicated pathological changes of the middle and inner ear structures which 

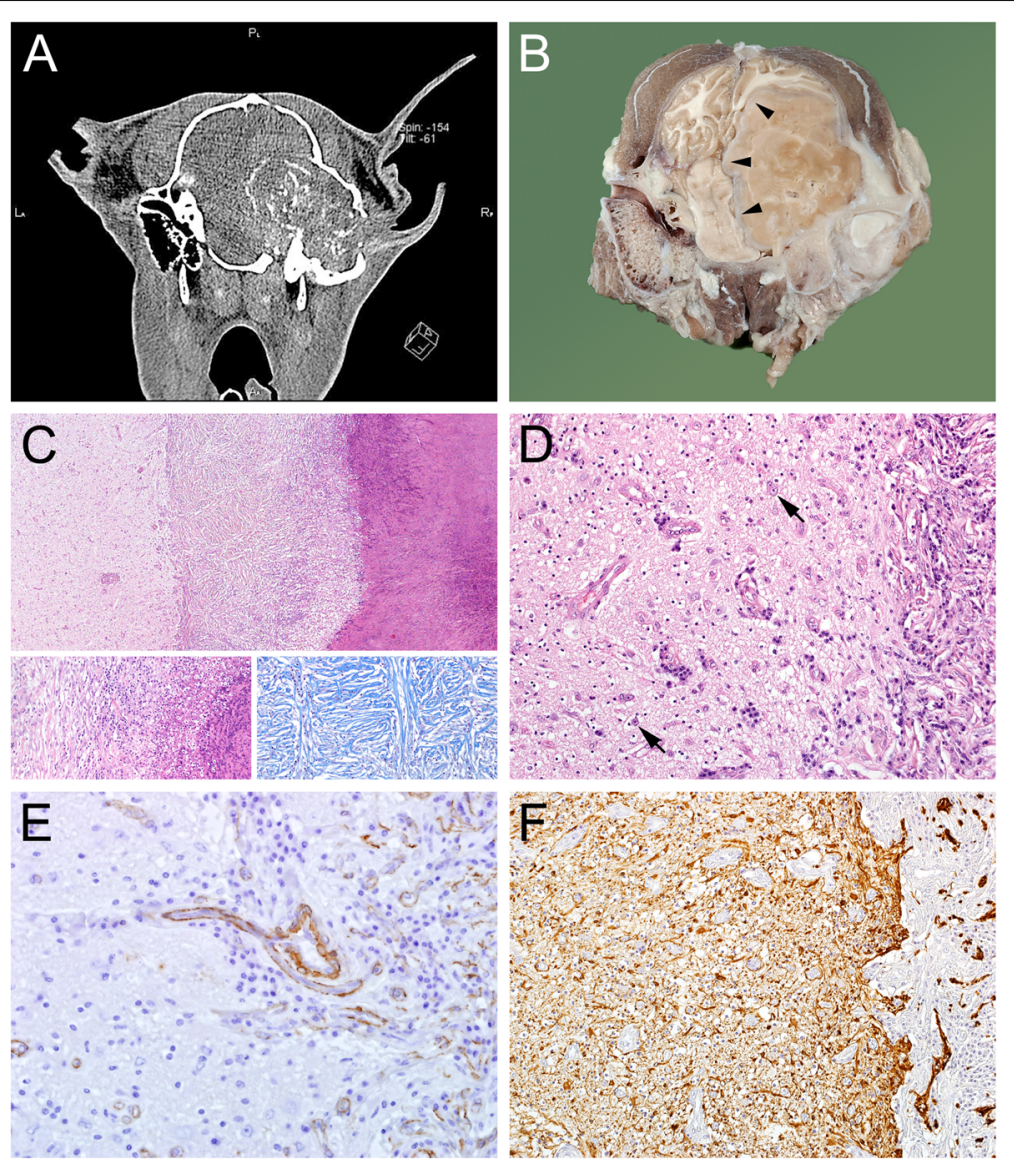

Fig. 4 a CT image of the head of the adult alpaca (soft tissue window). A partially mineralized soft-tissue mass originated at the level of the right ear and caused severe destruction of the temporal bone, midline shift of the brain and hydrocephalus (not seen on these planes), $\mathbf{b}$ corresponding macroscopic section to A of the cranium on the height of the ear canal, arrowheads depict the thin capsule of fibrous tissue around the abscess; A + B: the left ear canal is clearly visible, while the right ear canal and cranium is replaced by the abscess, causing severe midline shift and displacement of the brain to the left. $\mathbf{c}$ histologic overview of the brain abscess. Neuropil in the left third, abscess capsule in the middle third, necrotic center in the right third of the picture (HE-staining). Bottom left insert: detail of abscess capsule with granulation tissue and collagenous fibers to the left, degenerated neutrophils and necrotic cell debris to the right (HE-staining). Bottom right insert: Collagenous fibers (dark blue) in the abscess capsule (Masson's trichrome staining). $\mathbf{d}$ brain-abscess interface, abscess capsule to the right. Cerebral edema, gliosis and marked loss of neurons in the neuropil of the cerebral cortex, arrows: remaining neurons. Vascular proliferation and activation of endothelial cells and perivascular infiltration of lymphocytes (HE-staining). e brain-abscess interface, abscess capsule to the right. Infiltration of vessels from the abscess capsule into the neuropil (SMA-IHC). $\mathbf{f}$ severe diffuse astrogliosis (brown signal) in the neuropil of the cerebral cortex adjacent to the abscess capsule (GFAP-IHC)

were confirmed by $\mathrm{CT}$ examination. In cases showing unspecific neurological clinical signs CSF analysis can provide useful diagnostic hints and therefore the authors would recommend this procedure in such patients.

Llamas and alpacas are predisposed for developing otitis media/interna because of characteristic anatomic conditions such as a long and narrow external acoustic ear canal and a multicompartmental tympanic bulla $[3,9,11]$. Otitis media/interna can arise from otitis externa or is often presumed to be a consequence of ascending infection up the eustachian tube $[2,3]$. In the case presented, both ascending infection and otitis externa, could neither be confirmed nor ruled out as primary cause of the brain abscess due to the extensive lesions in the region of external, middle and internal ear structures and the chronicity of the process. However, due to the absence of pathological findings in other organs or structures otogenic origin seems very likely.

In general, bacteria, ectoparasites and foreign bodies, such as migrating plant awns have been described as causative agents for otitis externa, media and interna in SACs [3, 5, 11-14]. Listeria monocytogenes [13], Actinomyces spp., Streptococcus group D [14], Nocardia spp., and Proprionibacterium spp. have been isolated and associated with ear infections in SACs, but no consistent causative bacteria were described so far [11]. Psoroptes spp. is less common in SACs but primarily affects the ears $[3,15]$. From this alpaca only Chorioptes spp. were 
isolated from skin scrapings of the forelimbs, but the owner reported a history of mite infestation and subcutaneous antiparasitic treatment months before the alpaca was referred to the clinic. At this time no mite species differentiation was performed. Unfortunately, a complete ear canal stenosis was evident in this alpaca preventing further diagnostic measures at this site including biopsies.

Diagnostic imaging techniques, such as CT or MRI (magnetic resonance imaging) can be very helpful for antemortem diagnosis and are advantageous compared to radiography in cases demanding the visualization of medial and inner ear structures and the brain $[2,9,11$, $16,17]$. In this case it facilitated a profound diagnosis including the size and location of the abscess and the subsequent prognosis.

The histological lesions present in this patient were consistent with lesions described in a study regarding the neuropathological progression of brain abscess formation in dogs [18]. The authors of this paper divided the evolution of brain abscess formation into four stages based on histological criteria. In chronic cases with capsule formation they found a necrotic center, a peripheral zone of inflammatory cells and fibroblasts, and a collagenous capsule. The abscess was organized in the same way in our case as revealed by the histological samples analysed, although the necrotic center was very extensive. The capsule was quite thin, but contained collagenous fibers demonstrated by Masson's trichrome staining. This might be due to the long duration of abscess formation. Interestingly in our case a capsule could not be visualised by $\mathrm{CT}$ examination. It is known that in $\mathrm{CT}$ scans of individual patients the texture of the abscess can prevent differentiation between the central core of the abscess, the capsule and the surrounding brain tissue [16].

The formation of osteoid in the region of bone resorption was interpreted as callus formation and likely an attempt of tissue repair. Reactive astrocytes, gliosis and cerebral edema were also present in this alpaca as have been described in dogs [18]. The cerebral edema is most likely of vasogenic origin as a consequence of the physical breakdown of the blood brain barrier. Vascular proliferation and activation of endothelial cells was present in some regions of the neuropil adjacent to the abscess. Furthermore some vessels seemed to infiltrate the neuropil from the abscess capsule. This might be a reaction of the neuropil to the expansion and duration of the process. It also correlates to the lesions described in dogs with chronic brain abscesses, in which a layer of neovascularity in the periphery of the abscess associated with continuing inflammation of the surrounding neuropil was present [18]. As shown by immunohistochemistry activation of astrocytes and gliosis developed in the surrounding neuropil due to the chronicity of the process. This is a typical reaction pattern of the brain, which is generally found in areas surrounding severe focal lesions, areas responding to chronic neurodegenerative triggers or infections [19].

Based on the findings of gross examination of this alpaca an otitis can be assumed as primary disease in the case presented. As known from the case history mite infestation was detected and treated by the owner a prolonged time before referral. It can be hypothesized that in this alpaca chronic mite infestation of the external ear canal led to damage of the tympanic membrane and to secondary bacterial infection of the inner ear structures, and finally to brain abscessation.

In general, treatment of intracranial abscesses is difficult and involves parenteral administration of antimicrobial drugs such as penicillin, florfenicol or oxytetracycline, using high dosages in order to reach efficacy inside the abscess [3]. Often antimicrobial treatment alone is inadequate and a surgical approach has to be performed. Craniotomy with excision of a brain abscess was performed successfully in a 1 month old cria [6]. Nevertheless prognosis is described fair to guarded [3]. In this case craniotomy with excision of the abscess had not been feasible due to the huge size of the abscess, and even surgical drainage, a technique that is described for removal of abscesses adjoining the cranium $[3,6]$ would have failed due to size and gelatinous consistency.

To our knowledge this is the first case report describing the incidence of otogenic brain abscess formation in SACs, the clinical signs, diagnostic procedures and pathological findings.

\section{Abbreviations}

CNS: Central nervous system; CSF: Cerebrospinal fluid; CT: Computed tomography; GFAP: Glial fibrillary acidic protein; HE: Hematoxylin and eosin; IHC: Immunohistochemistry; MRI: Magnetic resonance imaging; PCR: Polymerase chain reaction; SACs: South American camelids; SMA: Smooth muscle actin

\section{Acknowledgements}

The authors thank the technicians of the Clinical Pathology Platform and the team of the Clinic of Anesthesiology and Intensive Care for performing anesthesia during computed tomography.

\section{Authors' contributions \\ SF and AD supervised inpatient care of the alpaca at the clinic and performed physical examination of the alpaca and SF performed otoscopic and ultrasonographic examination, AD and SF prepared the manuscript, SH performed necropsy and pathohistological examination, MG performed computed tomography. All authors read and approved the final manuscript.}

\section{Funding}

Not applicable.

\section{Availability of data and materials}

All data generated or analysed during this study are included in this published article.

Ethics approval and consent to participate Not applicable. 


\section{Consent for publication}

Consent was obtained in written form from the owner of the alpaca for publication of this case report and related images.

\section{Competing interests}

The authors declare that they have no competing interests.

\section{Author details}

${ }^{1}$ Clinic for Ruminants, Clinical Unit of Ruminant Medicine, University of Veterinary Medicine Vienna, Veterinärplatz 1, 1210 Vienna, Austria. ${ }^{2}$ Institute of Pathology, University of Veterinary Medicine Vienna, Veterinärplatz 1, 1210 Vienna, Austria. ${ }^{3}$ Clinical Unit of Diagnostic Imaging, University of Veterinary Medicine Vienna, Veterinärplatz 1, 1210 Vienna, Austria. ${ }^{4}$ Institute of Pharmacology and Toxicology, University of Veterinary Medicine Vienna, Veterinärplatz 1, 1210 Vienna, Austria.

Received: 17 December 2018 Accepted: 23 May 2019

Published online: 04 June 2019

\section{References}

1. Morin DE. Brainstem and cranial nerve abnormalities: listeriosis, otitis media/ interna, and pituitary abscess syndrome. Vet Clin North Am Food Anim Pract. 2004:20:243-73.

2. Whitehead CE, Bedenice D. Neurologic diseases in llamas and alpacas. Vet Clin North Am Food Anim Pract. 2009:25:385-405.

3. Cebra C, Gemensky-Metzler A. Disorders of the Neurologic System and special Senses. In: Cebra C, Anderson DE, Tibary A, Van Saun RJ, Johnson LW, editors. Llama and Alpaca Care. Medicine Surgery, Reproduction, Nutrition, and Herd Health. St Louis: Elsevier; 2014. p. 437-464.

4. Tsur I, Harmelin A, Dvir I, Yanai J. Meningoencephalitis and brain abscessation due to Escherichia coli in a 2 week old alpaca cria. Aust Vet J. 1996;74:437-8

5. Chigerwe M, Middleton JR, Pardo I, Johnson GC, Peters J. Spinose ear ticks and brain abscessation in an alpaca. J Camel Pract Res. 2005;12:145-7.

6. Talbot CE, Mueller K, Granger N, Jefferey ND. Diagnosis and surgical removal of brain abscesses in a juvenile alpaca. J Am Vet Med Assoc. 2007;231:1558-61

7. Hengrave-Burri I, Tschudi P, Martig J, Liesegang A, Meylan M. Neuweltkameliden in der Schweiz. II. Referenzwerte für hämatologische und blutchemische Parameter. Schweiz A Tierheilkd. 2005:147:335-43.

8. Braun U. Atlas und Lehrbuch der Ultraschalldiagnostik beim Rind.1st ed. Berlin: Blackwell Science; 1997.

9. Concha-Albornoz I, Stieger-Vanegas SM, Cebra CK. Computed tomography features of the osseous structures of the external acoustic meatus, tympanic cavity, and tympanic bulla of llamas (Lama glama). Am J Vet Res. 2012;73: 42-52.

10. Hardefeldt LA, Rylander H, Iskandar BJ, Ellison M, Peek SF. Diagnosis and surgical treatment of an intracranial cyst in an alpaca cria. J Am Vet Med Assoc. 2012;240:1501-6.

11. Galvan N, Middleton JR, Cook C, Britt LG, Kuroki K. Otitis interna, media, and externa with destruction of the left tympanic bulla and subluxation and septic arthritis of the left temporomandibular joint in an alpaca (Vicugna pacos). Can Vet J. 2013:54:283-5.

12. Fowler ME, Gillespie D. Middle and inner ear infection in llamas. J Zoo Anim Med. 1985;16:9-15.

13. Van Metre DC, Barrington GM, Parish SM. Otitis media/interna and suppurative meningoencephalomyelitis associated with listeria monocytogenes infection in a llama. J Am Vet Med Assoc. 1991;199:236-40.

14. Koenig J, Watrous B, Kaneps A. Otitis media in a llama. J Am Vet Med Assoc. 2001;218:1619-23.

15. Dawn EM. Brainstem and cranial nerve abnormalities: listeriosis, otitis media/ interna, and pituitary abscess syndrome. Vet Clin North Am Food Anim Pract. 2004;20:243-73.

16. Audigie F, Taprest J, George C, Didierlaurent D, Foucher N, Faurie F, Houssin $M$, Denoix JM. Magnetic resonance imaging of a brain abscess in a 10month-old filly. Vet Radiol Ultrasound. 2004:45:210-5.

17. Sumner JP, Mueller T, Clapp KS, Darien BJ, Forrest LJ, Colopy SA. Modified ear canal ablation and lateral Bulla osteotomy for Management of Otitis Media in an alpaca. Vet Surg. 2012;41:273-7.
18. Britt RH, Enzmann DR, Yeager AS. Neuropathological and computerized tomographic findings in experimental brain abscess. J Neurosurg. 1981;55: 590-603.

19. Sofroniew MV, Vinters HV. Astrocytes: biology and pathology. Acta Neuropathol. 2010;119:7-35.

\section{Publisher's Note}

Springer Nature remains neutral with regard to jurisdictional claims in published maps and institutional affiliations.
Ready to submit your research? Choose BMC and benefit from:

- fast, convenient online submission

- thorough peer review by experienced researchers in your field

- rapid publication on acceptance

- support for research data, including large and complex data types

- gold Open Access which fosters wider collaboration and increased citations

- maximum visibility for your research: over $100 \mathrm{M}$ website views per year

At $\mathrm{BMC}$, research is always in progress.

Learn more biomedcentral.com/submissions 